\title{
B6C3 Mouse
}

National Cancer Institute

\section{Source}

National Cancer Institute. B6C3 Mouse. NCI Thesaurus. Code C76182.

Derived from a cross between a C57BL/6 female and a C3H male, this hybrid strain is commonly used in the production of transgenic mice. B6C3F1 hybrid females are used as egg donors in the creation of transgenic mice as their breeding performance is robust and the pronuclei of their fertilized eggs are prominent, allowing for ease of microinjection. 